\title{
VALIDATION AND COMPARISON OF ANALYTICAL Q-BALL IMAGING METHODS
}

\author{
M. Descoteaux ${ }^{1}$, P. Savadjiev ${ }^{2}$, J. Campbell ${ }^{2,3}$, G.B. Pike ${ }^{3}$, K. Siddiqi ${ }^{2}$, R. Deriche ${ }^{1}$ \\ ${ }^{1}$ Odyssée Laboratory, INRIA/ENPC/ENS, INRIA Sophia Antipolis, France \\ ${ }^{2}$ School of Computer Science, ${ }^{3}$ McConnell Brain Imaging Center, McGill University, Montreal Canada
}

\begin{abstract}
Q-ball imaging (QBI), introduced by D. Tuch, reconstructs the diffusion orientation distribution function (ODF) of the underlying fiber population of a biological tissue. An analytical solution for QBI was recently proposed by several independent groups, using a spherical harmonic $(\mathrm{SH})$ representation of the input signal. The methods differ primarily in the way SH are estimated. In this paper we validate these methods and compare them against Tuch's numerical QBI on synthetic data, on a biological phantom and on a human brain dataset. We show that analytical QBI results in a speed-up factor of 15 over Tuch's QBI, while providing results that are in strong agreement. We also show that at the cost of slightly reducing angular resolution, QBI with Laplace-Beltrami regularization provides the strongest robustness to noise and the most accurate detection of fiber crossings.

Keywords: diffusion tensor imaging (dti), high angular resolution diffusion imaging (hardi), q-ball imaging (qbi), orientation distribution function (odf), regularization.
\end{abstract}

\section{INTRODUCTION}

Q-Ball Imaging [1] is a high angular resolution diffusion imaging (HARDI) reconstruction method that aids in the inference of fiber bundles with crossings, with advantages over diffusion tensor imaging (DTI). QBI seeks to reconstruct the diffusion orientation distribution function of water molecules in the underlying fiber population. There are other existing high order techniques in the literature such as those reviewed in $[2,3]$. Most such techniques lack a straightforward regularization process and are usually computed numerically, thus adding a computational burden.

Descoteaux et al. [4] have recently proposed an analytical solution to ODF reconstruction in QBI using SH and the Funk-Hecke theorem. Independently and with different derivations, Anderson [5] and Hess et al [6] have also developed essentially the same solution using SH. In [4], a LaplaceBeltrami (LB) regularization step is included in the estimation of the SH coefficients whereas Tikhonov (TKH) regularization is used in [6], while no regularization is used in [5].

In this article we first compare the performance of the analytical QBI method with LB regularization [4] against numerical QBI [1], considering computational complexity, running time, and robustness to noise. We also compare the method of [4] with those of [5] and [6] with regard to accuracy of fiber detection and limits on angular resolution. We conclude that the analytical QBI with LB regularization has the following advantages: 1) it is about 15 times faster than numerical QBI with ODFs that are in strong agreement and 2) at the cost of slightly reducing angular resolution with respect to $[5,6]$, the solution is robust to noise, improves fiber detection accuracy and reduces angular error between detected crossing fibers.

\section{METHODS}

\subsection{Analytical and Numerical QBI Implementation}

For the analytical solution, the signal is estimated at each of the $N$ gradient directions $i$ as $S\left(\theta_{i}, \phi_{i}\right)=\sum_{j=1}^{R} c_{j} Y_{j}\left(\theta_{i}, \phi_{i}\right)$, where $Y_{j}$ is the $j^{\text {th }}$ element of the SH basis and $R=(1 / 2)(\ell+$ $1)(\ell+2)$ is the number of terms in the basis of order $\ell$, choosing only even orders. Our implementation is based on a modified symmetric, real, orthonormal basis where a LB regularization approach (with parameter $\lambda$ ) is used to eliminate unnecessary higher order terms in the SH approximation [4]. In [6], Hess et al.'s implementation uses classical TKH regularization to obtain the $c_{j}$ coefficients at high orders $(\ell>4)$ and a simple unregularized solution for $\ell=4$. Spherical harmonics allow the simplification of the Funk-Radon Transform (FRT) by the Funk-Hecke theorem. The final ODF reconstruction, $\Psi$, is simply a diagonal linear transformation of the signal SH coefficients $c_{j}, \Psi(\mathbf{u})=\sum_{j=1}^{R} 2 \pi P_{\ell_{j}}(0) c_{j} Y_{j}(\mathbf{u})$, where $\ell_{j}$ is the order associated with $j^{t h} \mathrm{SH}$ basis element and $P_{\ell_{j}}(0)$ a Legendre polynomial.

Our implementation of Tuch's numerical QBI is taken from [7]. We choose $k=\sqrt{8 \pi N}$ integration points per equator to compute the Funk-Radon integral and set the angular width of the spherical Gaussian interpolation kernel to $\sigma=(3 / 2) \sqrt{2 \pi / N} .{ }^{1}$ This heuristic choice gives the best trade-off between accuracy and stability in our experiments.

\subsection{Data Generation and Real Data Acquisition}

First, we generate synthetic ODF data using the multi-tensor model $[4,8]$. We use a tensor profile with eigenvalues [3, 3,

\footnotetext{
${ }^{1}$ In Tuch [1], $k$ is set to $48, \sigma$ to $5^{\circ}$ and $N=755$ reconstruction points.
} 
17] $\times 10^{-4} \mathrm{~mm}^{2} / \mathrm{s}(\mathrm{FA}=0.8)$ and use complex Gaussian noise with standard deviation of $\sigma$, producing a signal with SNR $=1 / \sigma$. Then, the exact ODF is computed for $n$ fibers $[1,4]$. Second, we use a biological phantom dataset obtained from a $1.5 \mathrm{~T}$ scanner with 90 gradient directions, $2.8 \mathrm{~mm}$ isotropic voxels, and $b=3000 \mathrm{~s} / \mathrm{mm}^{2}$ [7]. Finally, we use a human brain dataset obtained from a 3T scanner with 99 gradient directions, $2 \mathrm{~mm}$ isotropic voxels, and $b=3000 \mathrm{~s} / \mathrm{mm}^{2}$.

\subsection{Computational Complexity Analysis}

Assuming the input data is of size $X \times Y \times Z \times N$, we have a diffusion signal vector of $N \times 1$ at each voxel. We let $k$ be the number of points on each equator over which the numerical FRT is computed. In analytical QBI, all methods $[4,5,6]$ have roughly the same computational complexity once $\mathrm{SH}$ coefficients are obtained. Letting $R$ be the number of elements in the SH basis, the analytical ODF reconstruction is $O(X Y Z N R)$ because of the $O(N R)$ matrix multiplication at every voxel, while the numerical ODF reconstruction is $O(X Y Z N k)$ because of the integration of $k$ equator points for each sampling direction $N$ at every voxel. Therefore, the difference in computational complexity between numerical and analytical QBI methods is between $R$ and $k$. For order $\ell=4,6,8, R=15,28,45$ and $k=48$, the theoretical speed-up factor is approximately 3,2 , and 1 , respectively.

\subsection{Experimental Validation}

Numerical and Analytical QBI Comparison To compare ODFs, we compute the mean Euclidean squared error between the estimated ODFs from numerical QBI [1] and from analytical QBI with $\mathrm{LB}$ regularization [4] on the rat and brain datasets. Then, to perform a quantitative evaluation of the ODF maxima, we use the ground truth orientations for the phantom from [9]. For each ODF dataset and for the DTI ellipsoids, the maxima are extracted. At each voxel, the smallest angular difference between the available maximum(a) and ground truth orientation(s) is recorded. The median and mean \pm standard deviation (std) orientation errors in degrees are then recorded.

Robustness to Noise In our synthetic data simulations we fix $b=3000 \mathrm{~s} / \mathrm{mm}^{2}, N=81$ (order 3 tessellation of the sphere), randomly choose $n$ between 1,2 , and 3 fibers and vary the SNR between 5 and 50 . For each SNR, we generate a set of 100 such raw HARDI datasets, estimate Tuch's numerical ODF and estimate our analytical ODF with and without regularization respectively. The optimal LB regularization $\lambda$ parameter for the $\mathrm{SH}$ approximation can be obtained from the L-curve numerical method, detailed in [8]. However, to avoid having to compute the optimal $\lambda$ for each HARDI profile, we set $\lambda=0.006$, a value experimentally found to be ideal for separating 1-fiber from 2-fiber distributions [8]. We use order $\ell=8$ and record the average Euclidean squared error between exact and estimated ODFs.

\begin{tabular}{c|c|c} 
dataset size (voxels) & $35 \times 17 \times 35 \times 90$ & $128 \times 128 \times 63 \times 99$ \\
\hline \hline Numerical QBI & $0: 13.59$ & $13: 27.12$ \\
Analytical QBI [4] & & \\
$\ell=4$ & $0: 01.27$ & $0: 40.42$ \\
$\ell=6$ & $0: 01.61$ & $0: 53.06$ \\
$\ell=8$ & $0: 02.09$ & $1: 49.37$
\end{tabular}

Table 1. A comparison of running times in minutes:seconds.

Fiber Detection and Angular Resolution It is generally assumed that the fiber directions are simply given by the local maxima of the normalized [0,1] ODF, where the function surpasses a certain threshold (here, we use 0.5). This relation can be used to extract local fiber orientation estimates for comparison with an estimated ground truth. To evaluate fiber detection differences between analytical QBI with LB [4], TKH [6] and no regularization [5] in ODF estimation, we test on noisy synthetic HARDI data with SNR 10, 2 orthogonal fibers, varying estimation order $\ell$ and $b$-factors of 3000 and $1000 \mathrm{~s} / \mathrm{mm}^{2}$. We generate 1000 such HARDI data separately, estimate ODFs with and without LB and TKH regularization, count the number of times we correctly detect 2 ODF maxima, and report the percentage and average angular error \pm standard deviation in degrees over all trials.

Finally, we perform a numerical experiment to evaluate angular resolution limitations of the ODF reconstruction methods. We generate noise-free synthetic HARDI profiles for 2 fibers and for $b$-factors of 3000 and $1000 \mathrm{~s} / \mathrm{mm}^{2}$. Then, we vary the crossing angle between fibers to determine the critical angle at which only a single maxima is detected instead of two. We report this critical angle as the angular resolution limit of the estimation.

\section{RESULTS AND DISCUSSION}

Running Time Comparison Tbl. 1 shows that analytical QBI is up to 15 times faster than Tuch's numerical QBI in practice. Computation is performed on a single processor, $3.4 \mathrm{GHz}, 2$ GB RAM machine. The speed-up is mainly due to the interpolation kernel width of Tuch's approach (not considered in the complexity analysis), which adds a constant number of operations (4 to 7 in practice) at every equator point.

Numerical and Analytical QBI Comparison First, Fig. 1 shows that Tuch's numerical QBI and our analytical QBI are qualitatively almost identical after min-max normalization [1]. Moreover, the mean Euclidean squared difference between the ODFs leads to $0.55 \pm 0.17 \%$ difference. Similarly, the overall shapes of the ODFs reconstructed from the brain dataset are nearly the same with $0.68 \pm 0.23 \%$ difference, confirming the strong agreement. Finally, Tbl 2 shows that Tuch's numerical QBI and analytical QBI with LB regularization yield essentially the same angular error while significantly reducing 


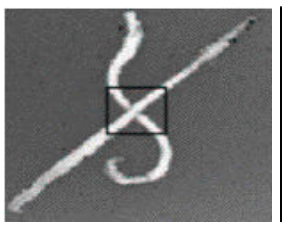

T1-weighted

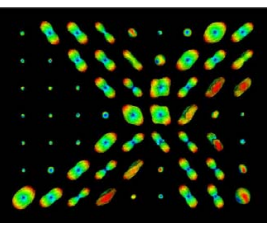

Tuch [1] QBI

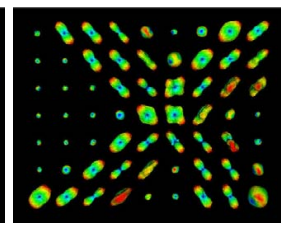

analytical QBI [4]
Fig. 1. Numerical and analytical QBI are almost identical.

\begin{tabular}{l||c|c}
\multicolumn{1}{c||}{} & \multicolumn{2}{c}{ Comparison with ground truth } \\
& median & mean \pm std \\
\hline analytical QBI [4] & $12.20^{\circ}$ & $15.94 \pm 15.32^{\circ}$ \\
numerical QBI [1] & $12.19^{\circ}$ & $15.94 \pm 15.40^{\circ}$ \\
DTI & $15.2^{\circ}$ & $19.4 \pm 16.2^{\circ}$
\end{tabular}

Table 2. Analytical and numerical QBI yield the same angular error while reducing the errors obtained from the DTI.

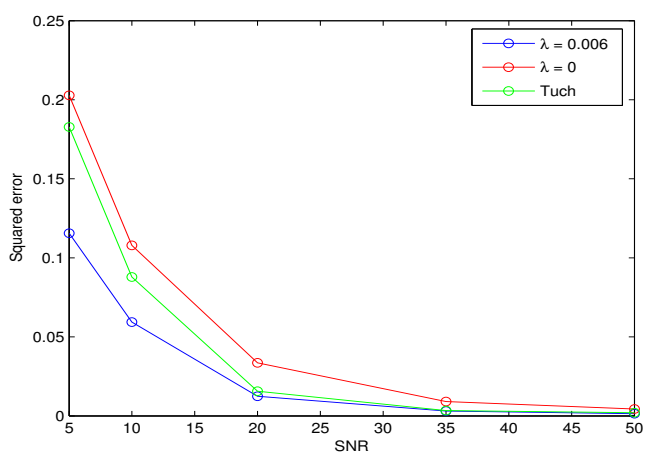

Fig. 2. LB regularized Analytical QBI is more robust to noise.

the errors obtained from DTI.

Robustness to Noise Fig. 2 shows that the LB regularization reduces ODF estimation errors using the simulation described in Section 2.2. As expected, the error decreases when the noise level decreases, from more than $12 \%$ for a noisy signal to less than $1 \%$ for high quality data. It is also important to note that for high quality data, numerical QBI and analytical QBI with and without regularization are almost identical whereas for noisy data, the analytical QBI with regularization $\lambda=0.006$ performs best, while numerical QBI is better than analytical QBI without regularization $(\lambda=0)$.

Fiber Detection and Angular Resolution Fig. 3 shows that LB regularized ODFs remove small perturbations due to noise that can create false maxima in ODFs estimated with and without TKH regularization. In particular, in this example there are 3 maxima detected for TKH and no regularization at $\ell=8$ and also an angular error made on the detected maxima at $\ell=6$. Tbl. 3 confirms the observation made in Fig. 3: LB regularized ODFs improve the detection of cross-
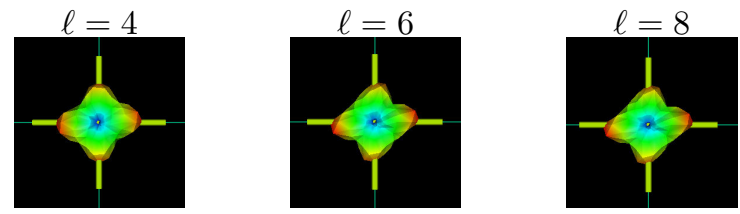

Laplace-Beltrami regularization
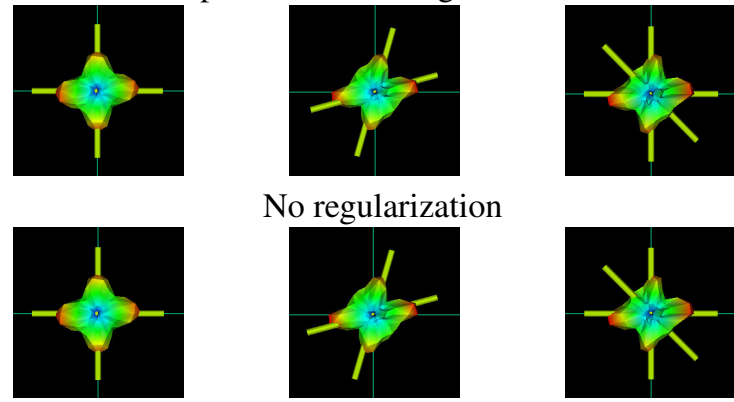

No regularization
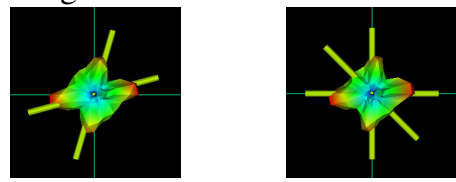

Tikhonov regularization

Fig. 3. LB regularization decreases perturbations due to noise that can create false maxima and maxima with angular error.

ing fibers while reducing angular error as calculated from the maxima of the ODF. In the case there were more than 2 maxima detected, the error was estimated on the two closest ODF maxima to ground truth. With LB regularization the detection is nearly perfect at $b=3000 \mathrm{~s} / \mathrm{mm}^{2}$ and above $88 \%$ at $b=1000 \mathrm{~s} / \mathrm{mm}^{2}$ for all orders whereas the detection dramatically decreases for high order estimations $\ell=6,8$ for TKH and no regularization. Tbl. 3 shows also that LB regularization reduces the average angular error as calculated from the ODF maxima detected and their ground truth. Overall, orthogonal fibers are detected accurately by all methods for $\ell=4$ even at the lower $b$-value. The approximation is smooth enough so that effects due to noise are reduced. LB regularization gives the best results for higher order ( $\ell$ 's $>4$ ).

The behavior of the ODFs in Fig. 3 and the improved performance in Tbl. 3 due to the LB regularization are expected. High-order modeling errors due to noise are avoided while minimally altering the lower order coefficients involved in the description of the ODF. The TKH regularization used in [6] mainly improves the numerical conditioning of the matrices. It is not designed to smooth the spherical functions as it perturbs the diagonal elements uniformly, which has the effect of adding the same weight to every eigenvalue. As seen in Fig. 3, this does not change the overall shape of the ODF and does not eliminate spurious peaks.

Finally, Tbl. 4 shows the critical separation angle of the analytical methods, i.e., the angle between 2 fibers under which only a single ODF maximum starts to be detected. As expected, it is harder to distinguish crossing fibers for lower $b$-values. In fact, there is approximately a $15^{\circ}$ gain in angular resolution when going from a $b$-value of 1000 to one of $3000 \mathrm{~s} / \mathrm{mm}^{2}$. For $\ell>4$ there is an improvement in angular 


\begin{tabular}{c|c|c|c|c}
$b$-factor & order $\ell$ & LB $(\lambda=0.006)[4]$ & without LB $(\lambda=0)[5]$ & Tikhonov [6] \\
\hline \hline \multirow{3}{*}{$3000 \mathrm{~s} / \mathrm{mm}^{2}$} & 4 & $99.9 \%, 2.1 \pm 5.4^{\circ}$ & $99.6 \%, 1.6 \pm 4.7^{\circ}$ & $99.6 \%, 1.6 \pm 4.7^{\circ}$ \\
& 6 & $99.6 \%, 2.8 \pm 6.1^{\circ}$ & $95.8 \%, 4.4 \pm 7.2^{\circ}$ & $95.9 \%, 4.2 \pm 7.0^{\circ}$ \\
& 8 & $99.4 \%, 2.5 \pm 5.8^{\circ}$ & $62.9 \%, 4.6 \pm 7.4^{\circ}$ & $63.1 \%, 4.5 \pm 7.2^{\circ}$ \\
\hline \multirow{3}{*}{$1000 \mathrm{~s} / \mathrm{mm}^{2}$} & 4 & $96.2 \%, 8.6 \pm 10.6^{\circ}$ & $96.1 \%, 7.1 \pm 8.9^{\circ}$ & $96.2 \%, 7.0 \pm 8.8^{\circ}$ \\
& 6 & $90.3 \%, 10.4 \pm 10.8^{\circ}$ & $69.4 \%, 11.9 \pm 10.1^{\circ}$ & $71.0 \%, 11.1 \pm 9.7^{\circ}$ \\
& 8 & $88.5 \%, 10.8 \pm 11.4^{\circ}$ & $23.4 \%, 11.3 \pm 10.9^{\circ}$ & $24.3 \%, 11.1 \pm 10.5^{\circ}$
\end{tabular}

Table 3. LB regularization improves the percentage of crossing fibers detected while reducing angular error.

\begin{tabular}{c|ccc|ccc} 
& \multicolumn{3}{|c|}{$b=3000 \mathrm{~s} / \mathrm{mm}^{2}$} & \multicolumn{3}{c}{$b=1000 \mathrm{~s} / \mathrm{mm}^{2}$} \\
order $\ell$ & Laplace-Beltrami & Tikhonov & $\lambda=0$ & Laplace-Beltrami & Tikhonov & $\lambda=0$ \\
\hline \hline 4 & $63^{\circ}$ & $60^{\circ}$ & $60^{\circ}$ & $75^{\circ}$ & $71^{\circ}$ & $71^{\circ}$ \\
6 & $59^{\circ}$ & $53^{\circ}$ & $54^{\circ}$ & $74^{\circ}$ & $68^{\circ}$ & $68^{\circ}$ \\
8 & $58^{\circ}$ & $53^{\circ}$ & $53^{\circ}$ & $74^{\circ}$ & $68^{\circ}$ & $68^{\circ}$
\end{tabular}

Table 4. Angular resolution of the ODF estimation with/without Laplace-Beltrami $(\lambda=0.006)$ and Tikhonov regularization.

resolution of roughly $5^{\circ}$ for all methods because higher order frequencies are included in the estimation of the signal. Lastly, TKH or no regularization has a better angular resolution than using LB regularization. In the LB case, the critical angle remains reasonable at about $5^{\circ}$ higher. This small decrease in angular resolution is due to smoothing of the higher frequency information by the LB operator. Hence, there is a trade-off between accuracy of fiber detection and the limit on angular resolution.

\section{CONCLUSION}

This paper carries out a validation of recent analytical QBI methods $[4,5,6]$ and a comparison against Tuch's numerical QBI [1], with several important findings. First, analytical ODF estimation using spherical harmonics offers the advantage that the solution for all directions is obtained in a single step, which makes it 15 times faster than numerical ODF estimation. Second, both numerical and analytical QBI reconstruct a diffusion ODF that agrees with ground truth from synthetic data and from a biological phantom, and on brain data. Finally, at the cost of slightly reducing angular resolution, solving for Laplace-Beltrami regularized spherical harmonic coefficients describing the input signal reduces perturbations due to noise, enabling a higher order approximation to be used, which in turn improves fiber detection.

\section{REFERENCES}

[1] D. Tuch, “Q-ball imaging," Magnetic Resonance in Medicine, vol. 52, no. 6, pp. 1358-1372, 2004.

[2] D.C. Alexander, An Introduction to Diffusion MRI: the Diffusion Tensor and Beyond, Springer, 2006.
[3] E. Ozarslan, T.M. Shepherd, B.C. Vemuri, S.J. Blackband, and T.H. Mareci, "Resolution of complex tissue microarchitecture using the diffusion orientation transform (dot)," NeuroImage, vol. 31, no. 3, pp. 1086-1103, 2006.

[4] M. Descoteaux, E. Angelino, S. Fitzgibbons, and R.Deriche, "A fast and robust odf estimation algorithm in q-ball imaging," in ISBI, Arlington, Virginia, USA, Apr. 2006, pp. 81-84.

[5] A.W. Anderson, "Measurements of fiber orientation distributions using high angular resolution diffusion imaging," Magnetic Resonance in Medicine, vol. 54, pp. 1194-1206, 2005.

[6] C.P. Hess, P. Mukherjee, E.T. Han, D. Xu, and D.B. Vigneron, "Q-ball reconstruction of multimodal fiber orientations using the spherical harmonic basis," Magnetic Resonance in Medicine, vol. 56, pp. 104-117, 2006.

[7] J.S.W. Campbell, K. Siddiqi, V.V. Rymar, A. Sadikot, and B.G. Pike, "Flow-based fiber tracking with diffusion tensor q-ball data: Validation and comparison to principal diffusion direction techniques," NeuroImage, vol. 27, no. 4, pp. 725-736, Oct. 2005.

[8] M. Descoteaux, E. Angelino, S. Fitzgibbons, and R. Deriche, "Apparent diffusion coefficients from high angular resolution diffusion imaging: Estimation and applications," Magnetic Resonance in Medicine, vol. 56, pp. 395-410, 2006.

[9] P. Savadjiev, J. S. W. Campbell, B. G. Pike, and K. Siddiqi, "3d curve inference for diffusion mri regularization and fibre tractography," Medical Image Analysis, vol. 10, pp. 799-813, 2006. 